Trans

continentales
Transcontinentales

Sociétés, idéologies, système mondial

$12 / 13 \mid 2012$

Marchés de l'art émergents

\title{
À propos de Pékin 798 ?
}

Propos recueillis par Catherine Choron-Baix et Franck Mermier

\section{Marc Abélès}

\section{OpenEdition}

\section{Journals}

Édition électronique

URL : http://journals.openedition.org/transcontinentales/1315

DOI : 10.4000/transcontinentales. 1315

ISBN : 978-2-7351-1599-0

ISSN : 1775-397X

Éditeur

Editions de la maison des sciences de l'homme

\section{Référence électronique}

Marc Abélès, «À propos de Pékin 798 ? », Transcontinentales [En ligne], 12/13 | 2012, document 2, mis en ligne le 30 août 2012, consulté le 08 septembre 2020. URL : http://journals.openedition.org/ transcontinentales/1315; DOI : https://doi.org/10.4000/transcontinentales.1315

Ce document a été généré automatiquement le 8 septembre 2020

Tous droits réservés 


\title{
À propos de Pékin 798 ?
}

\author{
Propos recueillis par Catherine Choron-Baix et Franck Mermier
}

\author{
Marc Abélès
}

\section{NOTE DE L'ÉDITEUR}

Marc Abélès dirige depuis 2012 le Centre Franco-Argentin des Hautes Etudes de Buenos Aires.

Normalien, Marc Abélès est titulaire d'un doctorat de 3e cycle et d'un doctorat d'État en anthropologie. Entré au CNRS, il a été membre du Laboratoire d'Anthropologie Sociale de 1979 à 1995. De 1990 à 1998, il a siégé au Comité national du CNRS. Il a dirigé le Laboratoire d'anthropologie des institutions et des organisations sociales qu'il a contribué à créer (1995-2009). Il a été directeur de de l'Institut interdisciplinaire d'anthropologie du contemporain (IIAC, CNRS-EHESS) de 2010 à 2012. En 2005, il a été élu directeur d'études à l'EHESS où son enseignement traite de l'anthropologie des institutions. Il a été visiting scholar et professeur invité à Brown University, Stanford University, New York University Boston University, Universidad de Buenos Aires. Marc Abélès a consacré ses premiers travaux, sous la direction de Claude Lévi-Strauss, aux pratiques politiques d'une société d'Ethiopie méridionale (Ochollo). Par la suite il a mené des recherches sur le politique et les institutions dans les contextes français et européen en reprenant plusieurs questions abordées à propos des Ochollo. L'élection, les pratiques d'assemblées, la symbolique politique sont au coeur de ses travaux sur la vie politique en Bourgogne (Jours tranquilles en 89, 1989), sur les rituels de François Mitterrand (Anthropologie de l'État, 1990), sur l'Assemblée nationale (Un ethnologue à l'Assemblée, 2000), sur les avatars de la représentation politique (L'Echec en politique, 2005) et sur le Parlement européen (La vie quotidienne au Parlement européen, 1992). En 1993, à la demande de la Commission européenne, il a dirigé une recherche anthropologique au sein de cet organisme. Plus récemment il a abordé la question de la globalisation dans deux perspectives différentes dans Politique de la survie (2006) où il traite du déplacement du politique et de l'émergence de nouveaux lieux de pouvoir, et dans Anthropologie de la globalisation (2008) où il analyse les conséquences 
épistémologiques des bouleversements produits par la globalisation et propose de nouvelles orientations pour l'anthropologie.

En 2011, Il a publié Pékin $798^{1}$, une étude sur le lieu le plus fameux de l'art contemporain à Pékin. Il nous livre là une réflexion originale sur les rapports de l'art, de la politique et du marché, dans la Chine à l'ère de la globalisation.

1 Transcontinentales: Il nous a semblé qu'il y avait dans votre livre de nombreuses allusions à votre histoire personnelle.

2 Marc Abélès : La première chose à dire est que n'étant ni un spécialiste de la Chine, ni de l'art contemporain, il peut sembler paradoxal que j'aie commencé à travailler sur ces questions. Je crois que lorsque je me suis rendu là-bas en 1998, j'ai été capté par un lieu, comme cela peut arriver parfois. D'emblée, le fait de me retrouver dans cet espace où coexistaient le monde ouvrier et l'art contemporain, un système capitaliste évolué d'un côté et, de l'autre, une atmosphère de travail, c'était assez frappant. Il y avait aussi l'architecture de cette usine construite dans les années 1950 par les Allemands de l'Est de l'école du Bauhaus. Tous ces éléments pris ensemble m'ont captivé. Et, comme je travaillais sur la question de la globalisation et en particulier sur celle de la dialectique entre le global et le local, cette situation était tout à fait propre à produire un travail sur ce genre de question. Voilà le point de départ, le contingent. Certes, au fur et à mesure de la progression de l'enquête, je rejoignais des choses assez personnelles et ce livre me tient à cœur parce que je m'y implique personnellement. Ce n'est pas une monographie ethnographique classique dans le sens où, tout au long du livre, je reste impliqué. C'est aussi un livre construit dans des formes de dialogues et d'interactions avec les gens qui se trouvaient dans cette usine à un titre ou à un autre, ouvrier, artiste ou galeriste. Chaque histoire individuelle, assez complexe et assez fascinante, me renvoyait à des questions personnelles. J'ai également beaucoup dialogué avec la personne qui était mon interprète, Mengchan Zhang, avec laquelle j'ai beaucoup travaillé et qui appartient à une génération de jeunes chinoises qui n'ont pas connu la Révolution culturelle, qui n'en ont pas la mémoire. Elle a, elle aussi, découvert, à partir de ce travail, une partie de son passé. Donc, tout cela est assez prenant. Il s'agit d'une découverte par quelqu'un qui est d'une génération très différente, de toute une zone qui jusqu'alors lui était complètement étrangère.

3 TC: Vous aviez publié un ouvrage sur l'anthropologie de la globalisation. Inaugurez-vous, dans ce livre, une méthode d'investigation qui mettrait en pratique les orientations de recherche présentées dans votre livre Anthropologie de la globalisation? On le voit bien, notamment, au sujet du lien entre le global et le local que vous avez mentionné.

M. A. : Effectivement, voilà une interrogation qui me paraît cohérente avec ce que j'essaie de faire, puisqu'une partie de l'Anthropologie de la globalisation ${ }^{2}$ concerne aussi une interrogation sur la manière de faire de l'anthropologie aujourd'hui. Les conditions sont très différentes de celles que j'ai connues quand j'ai commencé, à une époque où beaucoup de questions concernant, notamment, l'hybridation dans laquelle nous vivons aujourd'hui n'étaient pas encore posées. On peut assurément parler d'hybridation pour un lieu comme le 798, qui est à la fois une ancienne usine, un symbole de la classe ouvrière en Chine et, dans le même temps, comme le disent certains, un nouveau Soho, un lieu global complètement marchandisé. Aujourd'hui, quand on travaille sur le terrain, on est obligé d'étudier la complexité, car les lieux sont eux-mêmes des espaces denses. Du point de vue méthodologique, la question de savoir 
comment aborder cette complexité se pose. Walter Benjamin est un fil conducteur qu'on retrouve aussi d'ailleurs dans l'Anthropologie de la globalisation, en tout cas à la fin du livre. L'idée est de traiter le lieu comme un passage, de la même manière que Benjamin a essayé d'y réfléchir. J'appelle cela une méthode de déplacement. Plutôt que de prendre le lieu comme une sorte d'entité fermée sur elle-même, on en part pour s'ouvrir à une pluralité d'histoires. Et le travail que j'ai réalisé avec les gens en témoigne précisément : ce livre est en fait comme une écriture plurielle, dans le sens où $\mathrm{y}$ participent très activement des mémoires très différentes, très contrastées, des points de vue qui ne se rejoignent pas nécessairement, que l'écriture anthropologique doit prendre en compte. Même la façon d'aborder les gens, d'essayer, non pas de présenter un ensemble fini, homogène mais, au contraire, différentes ouvertures, me parait assez cohérent avec la manière dont on peut concevoir aujourd'hui l'anthropologie de la globalisation. Alors, dans ce sens, c'est un peu un exercice d'anthropologie de la globalisation. Cela s'inscrit tout à fait dans la continuité des grandes orientations de l'anthropologie et de l'ethnographie, qui permettaient d'avoir un lieu unique et, à partir de là, d'aller vers le multiple. Il ne s'agit d'ailleurs pas tant du multiple que de la pluralité de l'histoire. Je tente de trouver des points de raccord entre ces histoires et éventuellement des points de contradiction par rapport à ma propre histoire. C'est pour cela que je suis impliqué et que je considère qu'il est inutile de faire ce genre d'anthropologie sans l'être soi-même complètement, dans la mesure où il existe tout un rapport au politique qui me paraît évident dans ce lieu. Il s'agit d'un constat a posteriori parce que, sur le moment, on le voit comme un lieu d'art qui peut être traité comme tous les lieux d'art actuels, c'est-à-dire comme une friche industrielle, etc. Mais, au fil des rencontres avec les uns et les autres, je me suis aperçu qu'en fait, tout le rapport à ce lieu est politique puisqu'il met en cause à la fois des artistes voulant développer une certaine liberté de création et la direction de l'usine, l'administration de Pékin, le parti communiste, etc. Donc d'emblée, chacun se définit dans un discours politique qui peut être un discours politique sur l'art, un discours de l'art sur la politique, etc.

5 TC : Vous avez parlé de la figure du flâneur. Est-ce que le 798 est une galerie marchande mondialisée qui convertirait la figure du flâneur en un produit hybride, né du mélange instable de l'ici et de l'ailleurs? On est tenté de dire que cette figure du flâneur de Benjamin est convertie à travers ces nouveaux lieux mondialisés. Aussi, mais là n'est pas la question, l'imaginaire du flâneur au XIX siècle n'est pas le même que celui du flâneur aujourd'hui. Peut-on, d'ailleurs, encore utiliser cette notion de nos jours à propos de tels lieux ?

6 M. A. : La référence à Benjamin n'a pas été évidente, bien que je le cite dans Anthropologie de la globalisation et que je lui accorde l'importance qu'il mérite. Le plus curieux est que cette idée de rapprochement avec les Passages est venue presque après la fin du travail de terrain. Le 798 a toujours été pensé en termes de spectacle. Au cours de l'enquête, j'avais cela en tête, puisque bien avant qu'il n'existe cette marchandisation et que le lieu ne s'engage dans sa vocation artistique, il y avait une manière de le présenter comme la vitrine de quelque chose. En l'occurrence, celle du communisme triomphant. Au départ, c'est un peu une usine modèle. Une usine extrêmement belle construite avec beaucoup de soin. Je raconte tout cela dans le livre : la qualité de la construction, le fait d'orienter les pavillons de telle ou telle manière, qui en fait pour les artistes un lieu merveilleux. À l'intérieur, on trouvait toutes sortes d'activités culturelles et sportives, des crèches, qui le destinaient à une certaine élite. 
Mais c'était aussi une usine qu'on montrait, qu'on exhibait, comme une réussite de l'industrie à Pékin. Donc tout cela, à mon avis, en fait un lieu assez spectaculaire et symbolique. Et, aujourd'hui, à l'autre bout de la chaîne, il constitue le lieu de la marchandisation réussie. À la fois la marchandisation et la consommation, mais pas n'importe lesquelles. Les gens qui s'y rendent entrent aussi dans le spectacle des œuvres d'art partout présentes. Eux-mêmes participent à cette vie des œuvres puisqu'ils sont photographiés; il y en a dans la rue, on peut jouer, c'est un espace complètement ludique, un lieu qui n'en est pas un. Ce n'est pas une galerie comparable au Centre Pompidou, par exemple, ou aux galeries à New York. Il existe à l'intérieur un élément ludique qui capte très vite les gens. Ils y jouissent de formes de consommation variées avec tous ces cafés, la possibilité de s'acheter des babioles, tout en étant imprégnés de cette esthétique contemporaine qu'ils découvrent. Et c'est précisément là que l'on ressent le mieux l'hybride. Pour autant, ce n'est pas un lieu pour touristes, même si, depuis les Jeux olympiques de 2008, il est présenté dans tous les dépliants sur Pékin et que beaucoup y viennent. Mais en étant resté là-bas sur une assez longue durée et en y étant retourné à plusieurs reprises à des moments différents, je me suis rendu compte que la majorité du public est tout de même chinois. Beaucoup de gens viennent de province et de nombreux Pékinois s'y rendent les week-ends. La population s'est donc entièrement réapproprié ce lieu. Dans le même temps, il faut aussi s'accorder sur la question de l'hybridité que j'entends dans le sens que lui donne Homi K. Bhabha qui, lui-même, reprend les théories benjaminiennes sur la traduction et sur la façon de dire qu'il n'y a pas d'hybridité au sens d'une sorte de multiculturalisme ou de métissage. Il s'agit plutôt d'une certaine tension entre des éléments culturels pas nécessairement en harmonie les uns avec les autres. L'hybridité peut aussi être une forme de résistance, et c'est ainsi que j'entends en parler. Pour revenir à la question du flâneur, je dirais que ce n'est pas celle qui m'intéresse le plus. On peut certes dire que l'ethnologue en est un mais je ne pense pas que cela s'apparente à la notion du flâneur telle qu'elle est décrite et située historiquement par Walter Benjamin.

7 TC : Une question sur le lieu en lui-même : est-ce qu'il ne résulterait finalement pas de la relation qui pourrait exister - et que vous montrez très bien dans le livre - entre le développement du marché de l'art contemporain en Chine et celui de Pékin comme métropole mondiale ? Est-ce que le 798 n'est pas aussi pensé comme un équipement urbain, inscrit dans le développement de la ville en tant que métropole, et voué à une récupération marchande qui tend toujours à dépasser la force de contestation initiale présente chez les artistes? Ce lieu n'était-il pas destiné au départ à être un équipement urbain, un lieu de récupération marchande, qui aurait aussi besoin de cette force de contestation initiale? En fait, dans cette question se retrouve implicitement celle des acteurs. Quels sont les acteurs décisifs qui ont fait que ce lieu est devenu ce qu'il est ? Quelles sont justement leurs intentions quant à son émergence ? A-t-il été vraiment pensé comme tel ou est-ce le résultat d'un processus qui leur aurait en partie échappé ?

M. A.: C'est un peu les deux, et je l'explique dans le livre quand je reprends l'historique. Disons qu'au départ, il n'y a rien de véritablement pensé. Ce lieu n'est pas l'équivalent du 104, à Paris, où l'on transforme une friche en un espace d'expression artistique. Ce qui est drôle c'est que les Français de Pékin critiquent l'association. Ils disent que c'est une friche industrielle reconvertie. D'une part, ce n'est pas vraiment une friche industrielle, parce que encore une fois, les ouvriers sont toujours là et, 
d'autre part, cela n'a pas été programmé. Au début, on avait affaire, d'un côté, au problème d'artistes assez marginaux cherchant des lieux pour s'installer à des prix acceptables et, de l'autre, à la préoccupation de la direction de l'usine qui essayait de rentabiliser puisqu'il lui fallait payer les ouvriers et assainir ses finances : une certaine convergence apparaissait entre les deux situations. Par conséquent, l'idée de louer des locaux aux artistes ne gênait a priori personne. Dans un deuxième temps, il y a eu ces galeries, en particulier internationales, et là, c'est devenu un problème, un problème aussi politique. Que faire à partir du moment où le site devient public, un lieu d'exposition, dans un contexte politique qui n'est pas nécessairement favorable à ce genre de choses? Le conflit a débuté lorsqu'ils ont décidé d'organiser un festival d'art contemporain. Pendant toute cette période, les choses étaient d'autant moins programmées que l'espace restait marqué par cette marginalité. Des performances avaient déjà eu lieu dans des endroits hypermarginaux auxquels très peu de gens pouvaient accéder. Mais là, en revanche, cela commençait à devenir un phénomène de grande ampleur. L'initiative inquiétait d'autant plus les autorités qu'elle leur échappait, ce qui pouvait être interprété comme une forme de résistance. C'est aussi au terme d'un conflit, d'une négociation, que les choses ont basculé. À ce point est entré en jeu Huang Rui, un personnage intéressant qui anime à la fois la résistance et la contestation. Dans les années 1980, cet artiste a vécu très jeune déjà une situation très dure. Après la répression en Chine de son premier mouvement artistique, il part au Japon pendant assez longtemps. Il revient et, avec l'aide d'une galerie japonaise, il monte la première galerie à l'intérieur du 798, ce Tokyo Project. C'est donc ainsi que cela démarre, et qu'il prend un ascendant non seulement artistique mais aussi politique. À partir de là, peu à peu, se dessine un projet. Lorsqu'on parle aujourd'hui avec lui, on voit bien que ce n'est pas une reconstitution fictive. Il a très vite pensé le 798 comme partie intégrante d'un quartier qui joue un rôle dans l'urbanisme de Pékin. Et lorsque les autorités décidèrent de transformer le lieu selon un scénario alternatif qui visait à le supprimer afin d'en faire un lieu de high-tech, industriel, compte tenu de sa situation très favorable par rapport à l'aéroport, il refusa. Son argument, repris par d'autres, est qu'il faut défendre le patrimoine, arrêter de tout démolir. C'est au nom de la sauvegarde du patrimoine et non de l'art contemporain qu'il devait être préservé. Il s'agissait aussi de dénoncer les nombreuses erreurs qui avaient été faites dans l'urbanisme de Pékin. Aujourd'hui encore, il continue de penser le 798 dans le cadre d'un aménagement, ce qui rejoint des préoccupations actuelles, non seulement des autorités mais aussi des spéculateurs immobiliers. À ce stade, les choses sont assez perverses. Les spéculateurs immobiliers ayant évidemment le pouvoir, en ont compris la force d'attractivité et se sont mis à raser et à reconstruire des morceaux du quartier. Toute une zone d'ateliers d'artistes s'est donc développée, mais elle reste dans une grande précarité puisque récemment encore des ateliers ont été détruits pour laisser place à des immeubles de rapport. Comme dans beaucoup de métropoles, l'idée sous-jacente est que les quartiers d'artistes constituent une forme de valorisation urbaine. Nous sommes donc face à la question très complexe des enjeux de la planification de l'urbanisme à Pékin. Ce qui me fascine dans cette ville, c'est que, jusqu'à présent, il n'y ait pas de cohérence. Quand on s'y promène, on rencontre des zones hypersophistiquées du point de vue de l'urbanisme et d'autres complètement spontanées. Actuellement, une forme de planification semble se dessiner du fait de l'augmentation des prix du foncier, qui deviennent exorbitants, et du nombre important d'affaires en jeu. 
TC : Dans votre livre, vous établissez une relation étroite entre le développement du marché de l'art et l'histoire. Les formes artistiques contemporaines comme l'hyperréalisme, le Pop Art ou d'autres ont-elles permis, en Chine, d'aborder les figures du passé - ce que vous montrez très bien dans votre livre au sujet de Mao - en gommant leurs aspérités politiques et en utilisant des formes artistiques et des régimes d'expression contemporains? Ces nouvelles formes artistiques permettraient de "lisser» les aspérités politiques des figures du passé en les rendant lisibles au niveau mondial, mais aussi acceptables au niveau local.

10 M. A. : Je suis frappé par la forte présence de la figure de Mao à travers des formes d'art qui le tournent en dérision, mais une dérision relativement soft, peu violente. Ce travail esthétique sur la figure de Mao a dû évidemment en son temps beaucoup choquer les autorités chinoises. C'est allé assez loin et cela restait, dans le même temps, tout à fait récupérable pour les Occidentaux et le marché de l'art. Cela mettait en rapport l'art chinois et l'art contemporain, puisqu'on sentait très fortement une inspiration du Pop Art. Par ailleurs, dans le réalisme cynique, on voit qu'est proposée à travers des personnages non plus une opposition explicitement politique, mais plutôt une forme de réflexion sur l'absurde. Cette peinture de l'absurde met encore plus en évidence les contradictions du régime, son caractère insupportable, voire son côté décervelant, parce que les personnages sont absolument vides. L'autre école faisant pendant au réalisme cynique serait le Political Pop, une adaptation du Pop Art qui reste cependant créative dans la mesure où elle réinvestit des motifs chinois plus traditionnels. Cependant, cela reste très assimilable par les Occidentaux. Au fond, c'est ce type de peinture qui a permis une installation rapide sur le marché de l'art avec un important effet sur le plan politique. Le fait que cet art soit reconnu au niveau international a obligé les autorités chinoises à se repositionner par rapport à ce phénomène. Leur intelligence a été de reconnaître l'existence de l'art contemporain et de le valoriser sur le plan international. La récupération n'est donc pas strictement occidentale, elle est même apparue assez rapidement en Chine, quand ces créations ont eu leur place sur le marché au début des années 2000. Les Chinois ont vraiment pris le train en marche et en ont rapidement récupéré les bénéfices. Alors que cet art était connu mais totalement marginal, sa diffusion à l'étranger participe aujourd'hui au soft power. En s'implantant sur le plan international, les Chinois se montraient capables de laisser se développer un art subversif. C'est aussi cela, le 798, une vitrine de la possible subversion. Lorsqu'on y $\mathrm{va}$, on a parfois accès à des lieux et à des expositions réellement critiques. Ce qui entraîne par ailleurs des phénomènes de censure aux formes diverses, comme des coupures d'électricité dans une galerie, pendant plusieurs semaines, empêchant ainsi la présentation des œuvres. Là encore, c'est assez pervers. À mon avis, le fait de maintenir des ouvriers dans cette usine sert à montrer en permanence aux artistes et aux galeristes qu'ils ne sont pas les seuls à occuper cet espace et qu'il n'est pas colonisé par cette catégorie de la nouvelle bourgeoisie. Cette configuration est assez peu perçue par les touristes et les étrangers. Pourtant, sur place, c'est une des composantes essentielles du lieu.

11 TC : Ai Weiwei, qui vient de parler récemment à la télévision et sur les radios françaises notamment, est un peu un résultat de cette hybridité, si vous la concevez comme une forme de tension. Sa reconnaissance internationale et chinoise lui permet de parler et lui garantit une très relative protection. 
12 M. A. : Le cas d'Ai Weiwei est intéressant parce qu'il est un produit de la Révolution culturelle. Son père était un grand poète, très célèbre, il s'est fait laminerpar la Révolution culturelle. Il a donc passé son adolescence dans ce contexte et a forgé sa personnalité dans une certaine résistance aux autorités, à un certain conformisme. Il est parti assez rapidement aux États-Unis où il s'est formé. Ensuite, il est revenu. Mais sa position a été assez souvent ambivalente puisqu'il était à la fois un artiste totalement contestataire, il faisait des performances inouïes à l'époque, et il est en même temps l'architecte du stade olympique de Pékin, le «nid d'oiseau ». Il bénéficiait donc d'une certaine tolérance du régime. Le fait qu'il ait pu, pendant un certain temps, prendre des positions assez violentes sans être inquiété, prouve qu'il bénéficiait de protection. En toile de fond, il y avait aussi des choses qu'on ne saisit évidemment pas de l'extérieur : des conflits actuels qui étaient en train de changer la direction du parti communiste. La répression subie par Ai Weiwei était, pour une partie des clans du régime, une manière de poser les limites de leur tolérance et de montrer, à un certain moment, qu'ils reprenaient le pouvoir. Cela témoigne du jeu existant dans l'opinion, non seulement à l'égard de cette nouvelle bourgeoisie mais aussi des classes populaires. Le régime s'appuie beaucoup sur la paysannerie et souhaite montrer que tout n'est pas acceptable, qu'on ne peut pas brader tout l'héritage de Mao, etc. Mais Ai Weiwei tient assez fermement ses positions car il a gagné beaucoup d'argent et il est un artiste reconnu au niveau international. Il pourrait donc tout à fait s'en aller. Il révèle ainsi un réel courage, mais il sait aussi qu'il n'est pas seul. Pour s'acquitter de l'impôt réclamé par l'État, les gens l'ont spontanément aidé, il a reçu un million de yuans. Il sait que beaucoup de gens l'entourent et puis il y a ses blogs, et Internet. Son mode de fonctionnement constitue l'une des grandes difficultés auxquelles doit faire face le régime actuel à Pékin. Au sein de la classe dirigeante, un certain nombre d'acteurs économiques et politiques savent que les choses vont continuer à évoluer dans ce sens et qu'il faut par conséquent passer une sorte de compromis historique. Dans cette perspective, le 798 est un lieu très intéressant parce qu'il en est l'image. La tension est permanente, mais il n'y a jamais de rupture totale ni dans un sens ni dans l'autre. C'est-à-dire que les autorités répriment jusqu'à un certain point, mais jamais trop malgré tout, et que les artistes vont jusqu'à un certain point, dans leur forme de résistance, tout en disposant de techniques et en génèrant des situations parfois assez ambivalentes, ce qui leur permet de rester des interlocuteurs et de garder une marge d'action. La question des acteurs est intéressante. Les artistes ne sont pas "largués", totalement passifs ou totalement dissidents, à la marge. Ils sont au cœur. Vu depuis Pékin, le 798 est à la fois totalement récupéré par l'Occident, par les autorités, et reste en même temps un lieu de contestation et de tension. Et cela se retrouve même à l'intérieur de chacun. C'est complexe. Sur le plan personnel, cela m'a fasciné parce que cela illustre tout le problème qu'on peut avoir avec des organisations comme le parti communiste et avec certains modes de gouvernance qui restent très puissants mais qui sont travaillés de l'intérieur...

13 TC : Une autre question qui prolonge un peu ce que vous venez de dire concerne le rôle de certains collectionneurs ou spécialistes occidentaux comme Guy Ullens. Comment les autorités, justement, négocient-elles avec la présence de ces genslà ? Car ils jouent tout de même un très grand rôle, ils initient un musée, ce qui est très important, sociologiquement et historiquement, pour la société chinoise. 
M. A. : Oui, mais il est vrai que le richissime homme d'affaires belge Ullens avait des liens d'affaires avec la Chine de longue date, ce qui lui a permis de constituer cette collection. En même temps, dans une certaine mesure, les autorités l'ont encouragé tout en lui imposant des limites. Par exemple, avoir une fondation en Chine est impossible, parce qu'elle sous-tendrait une subversion éventuelle, justement avec l'appui des fonds étrangers. Cette idée est capitale. Par conséquent, le statut juridique de son musée permet aux autorités de garder un contrôle permanent sur ce qui s'y passe. L'autre difficulté qu'il a rencontrée est l'accueil de ce projet par les Chinois. On lui a proposé des conditions intéressantes en matière de loyer mais, lorsqu'il a connu des problèmes financiers, il a quand même dû assumer, d'une année sur l'autre, pour cet énorme projet, des investissements considérables, alors qu'il n'était pas en mesure de disposer de toutes les ressources dont il aurait eu besoin. Et lorsqu'il s'est retrouvé dans cet équilibre assez fragile, on ne peut pas dire que les autorités l'aient énormément aidé. Aujourd'hui, on ne sait d'ailleurs pas très bien quel va être l'avenir de cet homme puisqu'il a dû vendre une partie de sa collection. Il s'est donc un peu désinvesti de cette entreprise-là. À présent, des Chinois la réinvestissent complètement. C'est là une des forces de l'art contemporain en Chine: le relais est assuré. Sur le plan international, de nos jours, le marché est un peu cyclique : il y a des modes, et l'art indien tend aujourd'hui à devancer l'art chinois. La crise a aussi représenté un manque à gagner pour ce dernier. En même temps, toute une classe de riches s'est mobilisée, des nouveaux riches qui ont compris l'intérêt qu'il y avait à investir dans l'art contemporain, et cela a regonflé de nouveau le marché. Ces gens ont donc pris le relais. Par exemple, en dehors du 798, une sorte de centre pour l'art contemporain, financé par un industriel chinois, est en train d'être créé. Celui-ci est en pleine construction, tout va très vite, preuve que ça continue à tourner. Mais quelqu'un comme Ullens aura joué un rôle majeur. Il existe d'autres cas, et ces personnalités ont fait le lien entre le marché de l'art occidental et la Chine. Mais il n'y a pas de « reconnaissance » du point de vue chinois. On laisse faire les artistes, on les encourage, dans une certaine mesure, mais il faut que tout reste quand même sous le contrôle des autorités.

\section{NOTES}

1. Marc Abélès, Pékin 798, Paris, Éditions Stock, 2011, 240 p.

2. Marc Abélès, Anthropologie de la globalisation, Paris, Payot, 2008 (Petite Bibliothèque Payot, 2012). 